\title{
Oral motor dysfunction after carotid endarterectomy: Challenges in diagnosing marginal mandibular nerve injury and effectiveness of rehabilitation
}

\author{
Seçilay Güneş®, Birkan Sonel Tur@ (1) \\ Department of Physical Medicine and Rehabilitation, Ankara University, Faculty of Medicine, Ankara, Turkey
}

Received: May 13, 2020 Accepted: July 23, 2020 Published online: March 04, 2021

\begin{abstract}
Carotid endarterectomy (CEA) is a frequently used surgical treatment for carotid artery stenosis. Cranial and peripheral nerve injuries are among the intraoperative complications. Diagnosing isolated injuries of the marginal mandibular branch of the facial nerve after CEA is challenging and leads to oral motor dysfunction that affects the patient's quality of life. Early diagnosis and treatment are valuable, since delayed diagnosis requires a surgical restoration of the affected nerve or muscle. In this article, we present a case of marginal mandibular nerve injury after CEA to increase the awareness on this complication and highlight the importance of rehabilitation for these cases, even in the chronic phase.
\end{abstract}

Keywords: Carotid endarterectomy, electrophysiology, facial nerve, marginal mandibular nerve, physical therapy, rehabilitation.

Carotid endarterectomy (CEA) is a surgical procedure for patients with carotid artery stenosis to prevent stroke. ${ }^{[1]}$ Cranial nerve injuries during operation are quite infrequent and n. vagus, n. hypoglossus are the most affected nerves. ${ }^{[2]}$ Facial nerve injuries can be due to a result of stroke after the CEA procedure or peripheral involvement in the surgical area. The most prominent hint for differentiating this involvement by physical examination is supra-nuclear lesions presenting with contralateral facial weakness of the lower face and peripheral lesions presenting with ipsilateral facial weakness. ${ }^{[3]}$

The marginal mandibular branch of the facial nerve (MMBFN), derived from the lower division of the facial nerve and extends below the body of the mandibula, supplies all the muscles of the lower lip. ${ }^{[4]}$ The injuries of this nerve after CEA are uncommon
(1.58\%) since the nerve is not so close to the operation field. However, it is important, as the symptoms of the patient can mimic central facial paralysis due to the spared zygomatic and buccal branches.

In this article, we present a rare case of MMBFN caused by CEA to increase awareness of this complication and highlight the importance of physical therapy and rehabilitation for these cases, even in the chronic phase.

\section{CASE REPORT}

A 67-year-old male patient was admitted to our Physical Medicine and Rehabilitation outpatient clinic with complaints of difficulty in opening his mouth, oral leakage during liquid food consumption, and speech difficulties. His medical history revealed that he was admitted to the neurology outpatient clinic

\footnotetext{
Corresponding author: Seçilay Güneş, MD. Ankara Üniversitesi Tıp Fakültesi Fiziksel Tıp ve Rehabilitasyon Anabilim Dalı, 06590 Çankaya, Ankara, Türkiye. e-mail: secilaygunes@ankara.edu.tr 

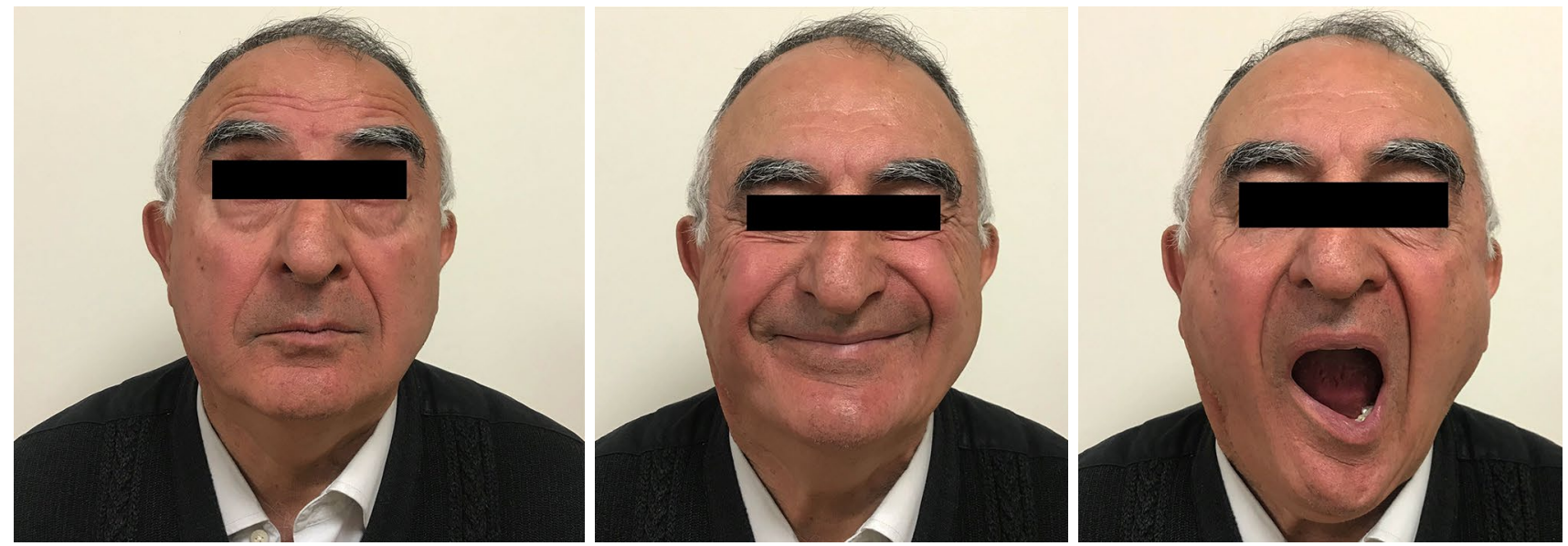

Figure 1. The patient could raise his eyebrows symmetrically. Nasolabial folds were symmetrical in the smiling position, but the dysfunction of the right depressor labii inferioris was clear, while he was opening his mouth.
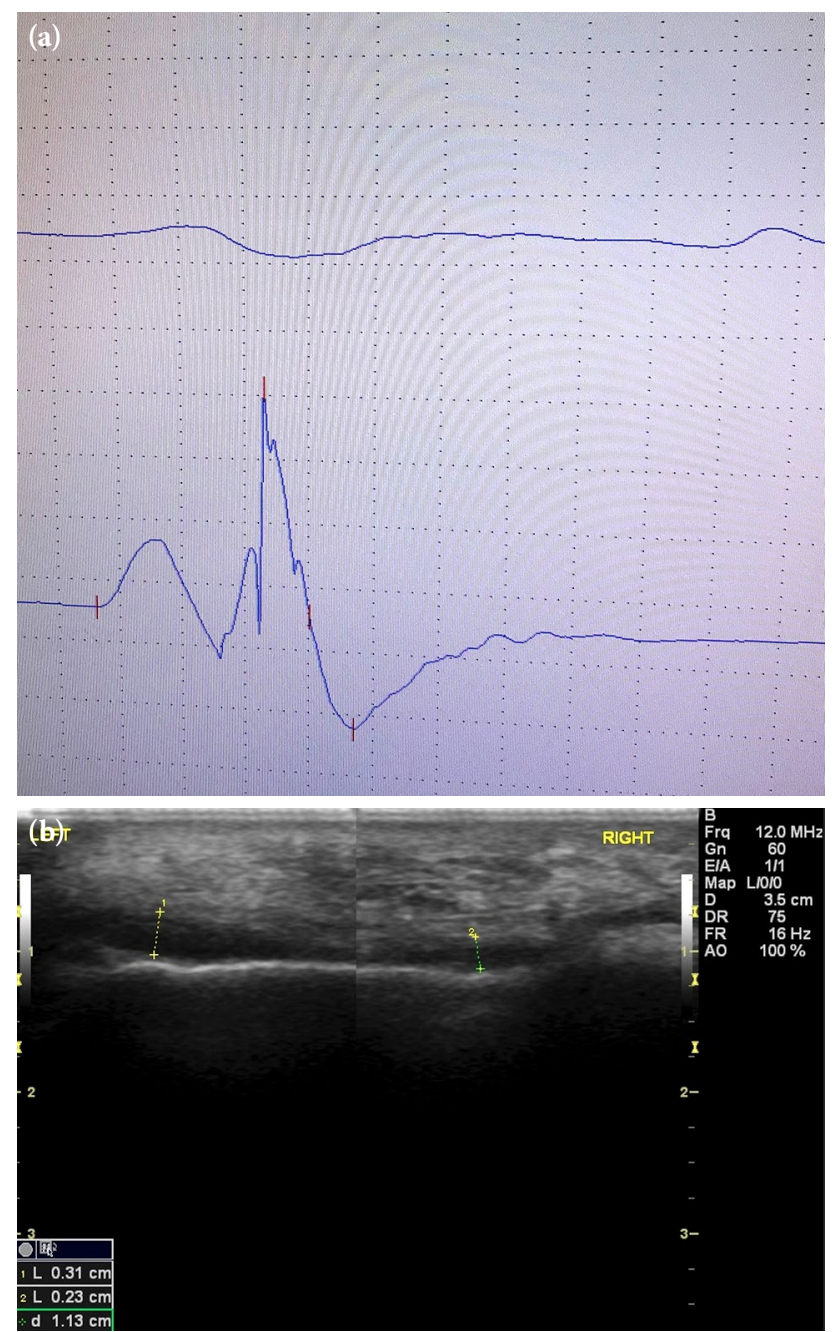

Figure 2. (a) The compound muscle action potentials of mentalis muscle were absent on the right side (upper) and normal on the left side (down). (b) An ultrasonographic view of mentalis muscle with a $12-\mathrm{MHz}$ linear probe on transverse position. with complaints of vertigo, the difficulty of speaking, and understanding 12 months prior. The complaints of the patient were found to be compatible with a transient ischemic attack. Doppler ultrasonography revealed 95\% stenosis in the internal carotid artery. Carotid endarterectomy and consequent patch angioplasty were performed by the cardiovascular surgery team and no postoperative complications were seen. After one month of the operation, his present complaints were started; however, these were not considered to be associated with operative complications.

On physical examination, he had an incision scar on his right sternocleidomastoid muscle. He could fully close his eyes, raise the eyebrows symmetrically. Nasolabial folds were symmetrical in the smiling position. However, the elevation of the right lower lip was evident, when the patient opened his mouth (Figure 1). Other neuromuscular examination findings were normal. An electrophysiological study of the facial nerve was conducted. Although the compound muscle action potential (CMAP) in the right orbicularis oculi by stimulating the zygomatic branch of the facial nerve was normal, CMAP of the right mentalis muscle by stimulating the MMBFN was absent (Figure 2a). Needle electromyography of the mentalis muscle revealed a single fiber activity at maximal voluntary activation. Sonographic evaluation of the mentalis muscle showed a decreased muscle thickness in the right side, compared to the left side (2.3 $\mathrm{mm}$ vs. $3.1 \mathrm{~mm}$, respectively) (Figure $2 \mathrm{~b}$ ). The patient was diagnosed with marginal mandibular nerve paralysis. A total of 15 sessions (five days a week for a total of three weeks), stretching and proprioceptive neuromuscular facilitation exercises, and massage were applied. Electrical stimulation was 
also administered to the lip depressor muscles for strengthening. After this program the fluid leak from his mouth and speech difficulty were fully recovered. A written informed consent was obtained from the patient.

\section{DISCUSSION}

Injuries of the MMBFN are rare and usually iatrogenic or traumatic. The physical findings assessed for routine facial nerve examination such as smiling, lifting eyebrows, and whistling are usually normal. However, when the patient is asked to open his/her mouth, which is an important maneuver for differentiating MMBFN injuries from the central facial paralysis, the affected lower lip becomes higher than the healthy side due to the dysfunction of the depressor labii inferioris muscle. ${ }^{[5]}$ Electrophysiological evaluation is important for the differential diagnosis. Normal values of the orbicularis oculi muscles evoked by stimulating the zygomatic branch of the facial nerve can exclude peripheral facial paralysis, but do not exclude MMBFN injuries. Additional stimulation of mentalis and depressor labii inferioris muscles should be kept in mind for an accurate diagnosis. Sonographic evaluation of the facial muscles is a new tool and quite helpful for showing muscle atrophy. The normal mean value of mentalis muscle in healthy individuals is are described as 3.0 (range, 1.4 to 4.2 ) $\mathrm{mm}^{.6]}$

Most of the cranial and peripheral nerve injuries are transient and recover within 6 to 12 months after CEA surgery. ${ }^{[2]}$ However, it is not clear whether these injuries are considered permanent or temporary in terms of time from injury. While surgical methods are preferred for permanent nerve damage in chronic patients, conservative methods are applied, if the paralysis is considered temporary. The primary goal of conservative treatment is to strengthen the affected lip muscles, thereby, improving eating and speaking function. Electrical stimulation is an important technique to activate the damaged neuromuscular system to achieve the lost function and it promotes nerve regeneration ${ }^{[7,8]}$ and it does not only stimulate the nerves, but also stimulates motion via muscle contraction which has a positive effect on the muscle activation and provides muscle strengthening. Surgical procedures usually consist of muscle and nerve transfers. ${ }^{[9]}$ Anterior belly of the digastric muscle and extensor digitorum brevis muscle transfers are preferable for restoring the lower lip depressor function. ${ }^{[10]}$ Re-innervation of the lower lip depressors with mini-hypoglossal or sural nerves is another option. ${ }^{[1]}$ Botulinum toxin injections can be used in patients who have cosmetic concerns, but refuses surgery. The goals of this procedure are to weaken the non-affected side depressor muscles and to improve the lower limp symmetry. ${ }^{[12]}$ However, botulinum toxin injections should be administered with caution, as this may increase lower lip dysfunction.

Although the effectiveness of surgical restoring procedures has been proven, surgical procedures are not always suitable for the geriatric population due to their associated comorbidities. This case, which was considered chronic nerve injury due to more than 12 months postoperatively, benefited from physical therapy methods, massage, and exercise. Despite asymmetry on his lips while he was opening his mouth continues, leakage during liquid food consumption returned to normal after rehabilitation, which was the primary goal for this patient.

In conclusion, injuries of the MMBFN are one of the rare complications after CEA and cause significant cosmetic and functional deficits which impair the quality of life of patients. As the zygomatic and buccal branches of the facial nerve are protected, it can be misdiagnosed as central facial paralysis. It still remains unclear what the maximum latency to start of rehabilitation is effective to achieve beneficial results. Physicians should increase the awareness of diagnosis and rehabilitation methods should be kept in mind as a favorable treatment option for patients with MMBFN paralysis before referring them to surgery, even if the time from injury is long.

\section{Declaration of conflicting interests}

The authors declared no conflicts of interest with respect to the authorship and/or publication of this article.

Funding

The authors received no financial support for the research and/or authorship of this article.

\section{REFERENCES}

1. Noiphithak R, Liengudom A. Recent Update on Carotid Endarterectomy versus Carotid Artery Stenting. Cerebrovasc Dis 2017;43:68-75.

2. Kakisis JD, Antonopoulos CN, Mantas G, Moulakakis KG, Sfyroeras G, Geroulakos G. Cranial nerve injury after carotid endarterectomy: incidence, risk factors, and time trends. Eur J Vasc Endovasc Surg 2017;53:320-35.

3. Ho ML, Juliano A, Eisenberg RL, Moonis G. Anatomy and pathology of the facial nerve. AJR Am J Roentgenol 2015;204:W612-9.

4. Rodrigues DCA, Andreo JC, Menezes DFL, Chinellato PT, Rosa-Júnior GM. Anatomy of the Facial Nerve and its Implication in the Surgical Procedures. Int J Morphol 2009;27:183-6. 
5. Moffat DA, Ramsden RT. The deformity produced by a palsy of the marginal mandibular branch of the facial nerve. J Laryngol Otol 1977;91:401-6.

6. Alfen NV, Gilhuis HJ, Keijzers JP, Pillen S, Van Dijk JP. Quantitative facial muscle ultrasound: feasibility and reproducibility. Muscle Nerve 2013;48:375-80.

7. Targan RS, Alon G, Kay SL. Effect of long-term electrical stimulation on motor recovery and improvement of clinical residuals in patients with unresolved facial nerve palsy. Otolaryngol Head Neck Surg 2000;122:246-52.

8. Ocak FDM, Tutar İ, Yıldırım A, Konuralp, N, Aydoğan A, Arslan BN, et al. Case report: Ramsay Hunt Syndrome, peripheral facial, paralysis, treated with electrostimulation. Turk J Phys Med Rehab 2005;51:111-3.
9. Tulley P, Webb A, Chana JS, Tan ST, Hudson D, Grobbelaar $\mathrm{AO}$, et al. Paralysis of the marginal mandibular branch of the facial nerve: treatment options. Br J Plast Surg 2000;53:378-85.

10. Conley J, Baker DC, Selfe RW. Paralysis of the mandibular branch of the facial nerve. Plast Reconstr Surg 1982;70:569-77.

11. Hanna DC. Discussion: Paralysis of the mandibular branch of the facial nerve. Plast Reconstr Surg 1982;70:577.

12. Butler DP, Leckenby JI, Miranda BH, Grobbelaar AO. Botulinum toxin therapy versus anterior belly of digastric transfer in the management of marginal mandibular branch of the facial nerve palsy: A patient satisfaction survey. Arch Plast Surg 2015;42:735-40. 